\title{
Meta
}

Journal des traducteurs

Translators' Journal

\section{MOUNIN, Georges (dir) (1993) : Dictionnaire de la linguistique, coll. "Quadrige ", no 153, Paris, Presses universitaires de France, XXXVII + 346 p.}

\section{Jean-Claude Boulanger}

Volume 39, numéro 3, septembre 1994

URI : https://id.erudit.org/iderudit/002112ar

DOI : https://doi.org/10.7202/002112ar

Aller au sommaire du numéro

Éditeur(s)

Les Presses de l'Université de Montréal

ISSN

0026-0452 (imprimé)

1492-1421 (numérique)

Découvrir la revue

Citer ce compte rendu

Boulanger, J.-C. (1994). Compte rendu de [MOUNIN, Georges (dir) (1993) :

Dictionnaire de la linguistique, coll. « Quadrige », no 153, Paris, Presses

universitaires de France, XXXVII + 346 p.] Meta, 39(3), 487-490.

https://doi.org/10.7202/002112ar d'utilisation que vous pouvez consulter en ligne.

https://apropos.erudit.org/fr/usagers/politique-dutilisation/ 
MOUNIN, Georges (dir.) (1993): Dictionnaire de la linguistique, coll, «Quadrige», $\mathrm{n}^{\circ} 153$, Paris, Presses universitaires de France, XXXVII $+346 \mathrm{p}$.

\section{Le dictionnaire}

Les années 1970 ont constitué une période charnière et faste en ce qui a trait à la publication des dictionnaires de linguistique de langue française. Aujourd'hui encore, ces répertoires demeurent les plus connus et les plus utilisés par les professeurs et les étudiants d'université. Pour se remémorer quelques titres très importants de la grande constellation des dictionnaires de cette période, il suffit de mentionner des œuvres-clés comme le Dictionnaire encyclopédique des sciences du langage (1972), le Dictionnaire de linguistique (1973), Le langage (1973), le Dictionnaire de didactique des langues (1976), le Vocabulaire de la linguistique (1976), Sémiotique: dictionnaire raisonné de la théorie du langage (1979). À ce sextuor dictionnairique, on ajoutera le Dictionnaire de la linguistique $(D L)$, originellement paru en 1974. Si l'on excepte quelques ouvrages plus thématiques et à caractère encyclopédique ainsi que les lexiques spécialisés, la décennie 1980 ne verra apparaître qu'un seul nouveau dictionnaire d'envergure, à savoir La grammaire d'aujourd' hui: guide alphabétique de langue française (1986). Par ailleurs, jusqu'à ce jour, seul le Dictionnaire encyclopédique des sciences du langage avait eu l'honneur d'une publication en format de poche. C'était en 1979. 
Le $D L$ devient à son tour accessible dans une collection de petit format, soit la célèbre collection «Quadrige». Cette réédition arrive à point nommé et elle produit un double effet: elle comble d'abord une lacune, car l'ouvrage d'origine était devenu introuvable, et elle ramène le coût d'acquisition à un niveau abordable pour la gent estudiantine, clientèle privilégiée pour ce type de dictionnaire.

La réédition dont on rendra compte n'est aucunement une refonte ou une mise à jour corrigée et augmentée du $D L$. Comme l'attestent la bibliographie, dont le plus récent titre est daté de 1973, et le directeur de la publication lui-même — il dit avoir ignoré la «mise en chantier» de trois ouvrages contemporains au sien, à savoir ceux «de Ducrot et Todorov, de Dubois et coll., de Pottier et coll.» (voir pp. XXIII-XXIV) —, à la virgule près, nous avons affaire au même texte que celui qui fut publié en 1974. En raison de sa remise à la disposition des utilisateurs, ce compte rendu du $D L$ prendra la forme de quelques rappels et de quelques observations critiques sur son contenu.

Comme beaucoup de ses semblables, le $D L$ est une cuvre collective, puisque, outre Georges Mounin qui a dirigé le projet, dix-neuf autres collaborateurs ont contribué à la rédaction des articles.

L'objectif fondamental du $D L$ est de fournir au public cible, formé d'étudiants, d'enseignants, de gens de culture..., un ouvrage de consultation efficace permettant de comprendre et de manier correctement les terminologies en usage dans différentes écoles de pensée linguistique actuelles. Le $D L$ n'a pas d'envergure encyclopédique, car il ne décrit pas de manière étendue tous les concepts qu'il recense. Il n'était pas dans les intentions des auteurs de préparer un manuel de linguistique cataloguant, en ordre alphabétique, quelques centaines de monographies sur les principales notions de la linguistique. Le $D L$ est donné comme un dictionnaire de mots, à savoir «celui qui réduit les définitions au minimum nécessaire pour comprendre le terme dans un contexte» (p. XXIII). Les responsables ont légitimement opté pour l'instrument de consultation rapide, efficace et ponctuel qui permet à l'utilisateur de poursuivre sa lecture ou sa recherche sans se perdre dans le labyrinthe des notions de la linguistique. Il constitue donc un ouvrage idéal pour les apprentis linguistes et les néophytes. Le programme auctorial recelait un objectif didactique fort bien réalisé dans le répertoire.

Le dictionnaire traite en priorité des termes de la linguistique et non pas de ceux qui sont relatifs à la grammaire. Les quelques unités de ce domaine qui y figurent sont perçues sous l'angle de l'analyse linguistique et non pas sous celui de l'analyse grammaticale traditionnelle. Il est à remarquer que pour la première fois, une place est faite à la terminologie de la linguistique appliquée. Le $D L$ est résolument synchronique. Il rend compte du vocabulaire utilisé il y a vingt ans et encore usuel aujourd'hui dans les écrits spécialisés de langue française. Il traite de la linguistique universelle, il ne se restreint pas à la seule linguistique française. En outre, la description n'a pas de visée normative. Selon Mounin, il ne revient pas à un dictionnaire de la linguistique de s'arroger le droit de choisir et de proclamer qu'il existe une seule bonne terminologie linguistique et de forcer tout le monde à entrer dans le moule. Dans une introduction bien étoffée, le responsable essaie de décrire le plus objectivement possible les causes historiques et théoriques qui ont conduit à l'inflation terminologique, inflation qu'il ne se prive pas de dénoncer malgré son intention de ne pas intervenir dans le processus de normalisation (voir le paragraphe 2 , ci-après).

La nomenclature répertorie 1306 termes (p. XXXVI, n. 1) appartenant à dix-huit sous-domaines de la linguistique générale courante, telles la dialectologie, la lexicologie, la phonologie, la sémantique, la syntaxe, etc., ainsi que la rhétorique et la versification, qu'on élève ici au rang de disciplines linguistiques. La microstructure des articles est simple. Elle est constituée de cinq rubriques fondamentales : l'entrée, l'indicatif de sous-domaine, 
la définition ou l'explication, un système de renvois à deux niveaux, les initiales du signataire de l'article.

La microstructure modèle est configurée de la manière suivante: 1 . L'entrée apparaît en caractères gras et avec une majuscule initiale, qui ne se justifie d'ailleurs aucunement. Cette rubrique est isolée des énoncés textuels et située en retrait des paragraphes explicatifs (exemples: Métathèse, Métonymie). 2. Le cas échéant, une ou des abréviations de sousdomaines de la linguistique précèdent le segment définitionnel ou les autres formes de commentaire (exemple : métaplasme appartient à la phonétique (phonét.), à la lexicologie (lexic.) ou à la rhétorique (rhét.)). L'absence de balise de domaine indique que le concept est relatif à la linguistique générale (exemples : philologie, phonétique). Mais tel n'est pas toujours le cas (voir mutation, pertinence, qui ne sont pas accompagnés de marques de domaine et qui relèvent nettement de la phonétique et de la phonologie, respectivement). 3. La rubrique sémantique définit le terme cible dans autant de champs d'application qu'il y a d'étiquettes thématiques. Les énoncés définitionnels sont généralement succincts et suffisamment explicites pour mener à une bonne saisie de la notion (exemples : lexicographie, mot-valise). Ils demeurent, pour la plupart, encore très actuels, quoique certains aient vieilli (voir par exemple la seconde définition de l'entrée lexicologie: «Désigne aussi la réflexion théorique sur les problèmes posés par l'élaboration des dictionnaires.»). Il est évident que cette vision n'est plus conforme à la réalité. La dictionnairique a suffisamment progressé pour mériter qu'on lui attribue une dénomination propre. D'ailleurs, le mot dictionnaire ne possède qu'une entrée-renvoi (dictionnaire: V. lexicographie, inverse). À l'occasion, la définition est enrichie d'une explication supplémentaire qui emprunte parfois des allures encyclopédiques (exemples: diachronie, racine). 4 . Le système de renvois est de type onomasiologique et il possède deux dimensions. D'abord, dans les énoncés explicatifs, les mots qui se retrouvent eux-mêmes à leur place alphabétique dans le dictionnaire sont suivis d'un astérisque (exemple: métaplasme, qui offre 17 renvois internes). Cette procédure n'est cependant pas systématique pour tous les mots d'un même article, pas plus qu'il n'y a de récursivité d'un article à l'autre. Ensuite, les renvois placés en fin d'article ou après le paragraphe définitionnel; cette rubrique est d'ordre synonymique (exemple : arrière: «Syn. : postérieure*, vélaire*.») ou d'ordre analogique, permettant ainsi de retracer des concepts apparentés (exemple: lexique: «V. monème, lexème, lexie, etc. V. aussi lexicographie, lexicologie.»). 5. Chaque article se termine par la signature de son rédacteur sous la forme de ses initiales. Georges Mounin n'a signé aucun article. Il paraît n'avoir fait que diriger cette recherche et en réviser les résultats. Curieusement, certains articles ne comportent aucune personnalisation (exemples : schlintement, schwa). Peut-être sont-ils l'œuvre de Mounin lui-même? L'avertissement au lecteur reste muet à ce sujet.

Les articles sont donc de structure simple et assez brefs tout en offrant le maximum d'information sur un concept de la linguistique. On regrettera cependant l'absence des catégorisations lexico-grammaticales. «On n'a pas donné non plus d'indications grammaticales: masculin, féminin, adjectif, etc.» (p. XXIV). Cette information est nécessaire pour les apprenants. Sinon, comment connaîtront-ils le genre des mots comme aphérèse, apocope ou realia, ou encore le statut de nom ou d'adjectif de mots comme homéonyme et homophone sans en référer à un autre dictionnaire? L'objectif de consultation ponctuelle perd ici de sa justification. Ce défaut amoindrit quelque peu la portée pédagogique de ce dictionnaire qui se veut d'abord un outil d'apprentissage.

Il reste toutefois que le $D L$ atteint son but premier - qui est aussi celui de tout dictionnaire thématique - , à savoir qu'il doit dissiper des malentendus et des malaises terminologiques tout en véhiculant des connaissances sur un sujet. L'exemple de l'entrée morphème est très éloquent à ce propos. On sait que son sémantisme varie selon que l'on 
lit Bloomfield, Hjelmslev, Troubetskoy, Vendryes ou Martinet. Il faut parfois un dictionnaire pour démêler cet écheveau. Dans cette optique, le $D L$ remplit adéquatement son rôle.

\section{Le $D L$ et la terminologie}

Le dictionnaire alphabétique est précédé d'un long texte de Georges Mounin intitulé : Introduction au problème terminologique (pp. VII-XXII) dont il vaut la peine de dire un mot. L'auteur traite abondamment du problème de l'inflation terminologique qui, à son avis, est dû à la néologie d'ignorance, c'est-à-dire à la création excessive de mots inutiles quand la langue de spécialité étudiée possède déjà des dénominations appropriées pour désigner un concept. Le chercheur aborde aussi une question complémentaire, soit celle de la contamination d'une terminologie par l'emprunt exagéré de termes à succès «aux sciences et aux découvertes scientifiques qui font du bruit» (p. XII). En dépit de ces mises en garde, Mounin est cependant conscient que contrecarrer la prolifération terminologique, la paralyser, l'unifier à tout prix, «ce serait immobiliser la recherche» (p. XIII). Toute tentative de standardisation unidirectionnelle lui apparaît utopique. Un dictionnaire idéal supposerait une langue morte. Il faut recommander la prudence devant la nouveauté, il ne s'agit nullement de l'interdire. Pour Mounin, il faut vivre avec le dilemme et, comme Meillet, il pense qu'une terminologie doit répondre à des aspirations contradictoires.

Cet article introductif est d'une très forte puissance évocatrice et il constitue un credo pour le terminologue. On est en présence d'une véritable pièce d'anthologie, d'autant que le sujet en est notre propre LSP, celle de la linguistique. Tout terminologue devrait en méditer chaque paragraphe. Il y a là quelques leçons à tirer car, même si elles furent consignées voilà vingt ans, elles gardent encore tout leur caractère de vérité scientifique et toute leur actualité. On ne saurait trop recommander la pratique assidue de ce texte afin de prendre quelques leçons fondamentales sur la profession de terminologue et sur celle de lexicographe, puisque, bien entendu, si les deux se ressemblent, elles divergent en beaucoup de points. Un bon terminologue n'est pas nécessairement un bon lexicographe, tant s'en faut. L'inverse est aussi vrai. Bien des terminologues ou des lexicographes, qu'ils soient profanes ou expérimentés, ou qu'ils se disent tels, trouveront ample matière à réflexion dans l'introduction du $D L$. En particulier, ils y découvriront l'importance de bien évaluer le programme des travaux projetés et les raisons qui conduisent les responsables à procéder à leurs recherches en se fondant sur l'idéologie qu'ils privilégient. Avant de critiquer ou de condamner sans examen approfondi et sérieux les intentions des auteurs d'une œuvre, mieux vaut s'assurer qu'on en mesure tous les tenants et les aboutissants. Toute auvre est le résultat d'un programme scientifique et elle s'inscrit dans un contexte spécifique. Telle est la leçon majeure que l'on retirera de ce véritable petit bijou mounien. Il faut lire, relire et approfondir cette très intéressante introduction qui n'a pas pris une ride et qui est l'œuvre d'un véritable professionnel et d'un chercheur expérimenté. Le décodage du dictionnaire qui suit ne saurait qu'en bénéficier. 